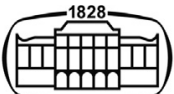

AKADÉMIAI KIADÓ

IMAGING

\title{
"Heart full of thrombi": Post COVID-19 \\ multisite thrombosis assessed by \\ echocardiography and pulmonary CT
}

\author{
I. MITEVSKA* $\odot$, E GRUEVA NASTEVSKA, E. KANDIC and \\ O. BUSLJETIK
}

Intensive Care Unit, University Clinic of Cardiology, Skopje, North Macedonia

Received: May 17, 2021 • Accepted: June 8, 2021

\section{CASE REPORT}

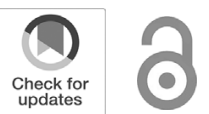

\begin{abstract}
Thrombosis in general, and especially venous thromboembolism (VTE) is one of the most common complications associated with COVID-19 infection. We present a 48 years old male patient with dyspnea and severe multisite post Covid-19 disease thrombotic complications, with pattern never seen before, that includes both ventricles, pulmonary arteries and peripheral vein involvement, assessed by echocardiography, vascular ultrasound and pulmonary CT angiography.
\end{abstract}

\section{KEYWORDS}

COVID-19, thrombosis, echocardiography, pulmonary CT angiography

\section{Case report}

A 48y old male (MD) was admitted to our intensive care unit with decompensated heart failure (HF). One month earlier he was hospitalized for "SARS-CoV-2" infection" confirmed by PCR test and pulmonary CT findings for COVID-19 pneumonia at the infective disease clinic. He had no previous history of cardiovascular disease (CVD). On admission he presented with dyspnea at rest and mild physical activity, tachyarrhythmia and signs of decompensated HF (NYHA III) with lower leg edema. Laboratory D-dimers values were $10.765 \mathrm{ngr} / \mathrm{mL}$ (normal values $500 \mathrm{ngr} / \mathrm{ml}$ ), NTproBNP $6700 \mathrm{pg} / \mathrm{ml}$ (normal values $125 \mathrm{pg}$ / $\mathrm{ml}$ ) and procalcitonin S 0,4 (normal values $0.15 \mathrm{ng} / \mathrm{mL}$ ), with other results within normal limits. In addition, urgent echocardiography revealed presence of multiple thrombi (7 in total), 5 in the left ventricle (LV) with different size and mobility (the largest LV thrombi had dimensions of $3.9 \mathrm{~mm} \times 2.4 \mathrm{~mm}$ ) (Fig. 1 - A and B; arrowheads). The ejection fraction of the LV was severely reduced with a global hypokinesia, left ventricular ejection fraction (LVEF) of $34 \%$, end diastolic volume $176 \mathrm{ml}$, end systolic volume $98 \mathrm{ml}$ at the admission with improvement of LV function at the discharge with LVEF of $45 \%$. Right ventricular (RV) function was also reduced with TAPSE 16, and TDI S' wave velocity $8 \mathrm{~cm} / \mathrm{sec}$, tricuspid regurgitation of $3.0 \mathrm{~m} / \mathrm{sec}$ and pulmonary artery systolic pressure of $37 \mathrm{mmHg}$. Also, there was large thrombus in the RV with dimensions $29 \times 32 \mathrm{~mm}$ and a smaller one with dimensions 13 x 10mm (Fig. 1 - B; arrowhead). Pulmonary artery was dilated $(27 \mathrm{~mm})$ with floating thrombus on the bifurcation (Fig. 1 - D), with a high risk of embolization. Inferior vena cava was with borderline dimensions $(20 \mathrm{~mm})$, with inspiratory collapse. CT angiography revealed pulmonary embolism $(\mathrm{PE})$, with thrombus extending towards the right pulmonary artery (Fig. 1 - D; arrowhead). Pulmonary CT scans showed multiple bilateral peripheral and central post COVID-19 fibrotic lesions (Fig. 1 - E; arrowheads). Venous Doppler ultrasound - Iliofemoral thrombosis: non compressive communis femoral vein, femoral superficial vein and non-occlusive thrombus in left popliteal vein. He was hemodynamically stable during hospitalization. Medical therapy for HF was started (perindopril 

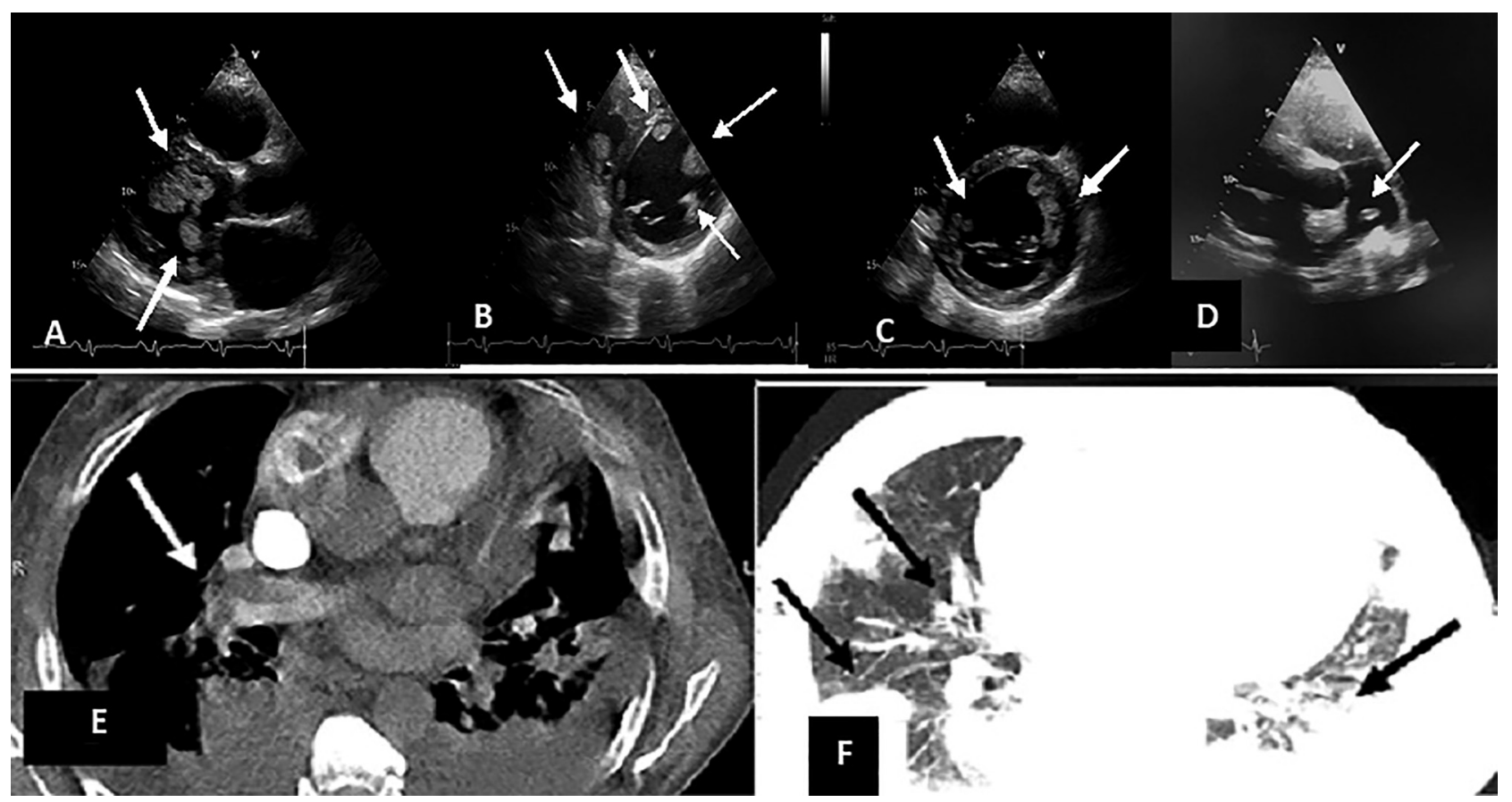

Fig. 1. Echocardiography, pulmonary CT and pulmonary CT Angiography findings: Echocardiography (A-D) showed large thrombmi in the left ventricle on the parasternal long axis view (PLAX), (A; arrowheads). Multiple thrombi in the right and left ventricle on the apical four chamber echocardiographic view (4C) (B; arrowheads). Multiple thrombi in the left ventricle on the short axis view (C; arrowheads). Short axis aortic valve view showing floating thrombus on the pulmonary artery bifurcation (D; arrowhead). Pulmonary CT angiography showing thrombus in the right pulmonary artery (E; arrowhead). Pulmonary CT showing multiple bilateral peripheral and central post COVID-19 fibrotic lesions (F; arrowheads)

$4 \mathrm{mg}$ od, carvedilol $6.25 \mathrm{mg}$ bid, spironolactone $50 \mathrm{mg}$ od, furosemide $125 \mathrm{mg} \mathrm{od}$ ) and anticoagulation with low molecular heparin $1.5 \mathrm{mg} / \mathrm{kg}$ bid for the first 3 days, then direct oral anticoagulant Rivaroxaban $15 \mathrm{mg}$ bid for the following 21 days. Confluent thrombus formation with decrease of thrombus number and mobility was noted on the control echocardiographic exam (Fig. 1 C; arrowhead). Performed thrombophilia analyses showed heterozygous mutations for factor V Leiden, prothrombin and plasminogen activator inhibitor PAI-1. Patient survived hospitalization and was discharged after 24 days with the medical therapy for heart failure and anticoagulation therapy with rivaroxaban $20 \mathrm{mg}$ od for three months with close follow up and possible extension of anticoagulant treatment up to 6 months. Coronary angiography, cardiac magnetic resonance (CMR) and complete testing for thrombophilias was scheduled to be additionally performed on outpatient basis.

\section{Discussion}

Coronavirus disease-2019, caused by the severe acute respiratory syndrome-coronavirus-2 (SARS-CoV-2), predisposes patients to thrombotic disease, due to excessive inflammation, platelet activation, endothelial dysfunction, and stasis. Early reports suggest incidences of deep vein thrombosis (DVT) and PE up to $30 \%$ in patients with COVID-19 disease $[1,2]$. Treatment of our patient was challenging because of the concomitant presence and complexity of the inflammatory consequences, severe hypercoagulable state and the myocardial dysfunction [2]. Bed site point of care ultrasound (POCUS) echocardiography is very useful imaging technique for fast assessment of LV function or PE indirect signs, which also provides possible answers for patient clinical instability [3]. Echocardiography enables detection and follow up of thrombus formations in the heart cavities and pulmonary artery. Vascular ultrasound and CT angiography are imaging methods that gives us an opportunity to assess and follow up thrombosis in different vascular beds [3]. The proposed mechanisms for COVID-19 disease-induced thrombosis include a cytokine-mediated diffuse microvascular damage, disease-specific hypercoagulable state, hypoxia, immobilization, diffuse intravascular coagulation and, in some cases, reactive thrombocytosis [4]. In the present case, the patient had elevated C-reactive protein $(45 \mathrm{mg} / \mathrm{l}$, normal values $6 \mathrm{mg}$ / 1) and D-dimer levels with no other risk factors for pulmonary embolism, thereby indicating a COVID-19 disease-related hypercoagulable state as a possible cause of multivesicular venous thrombosis including $\mathrm{PE}$. There are studies indicating that patients with LV thrombosis have significantly higher Creactive protein (CRP) values than the non-thrombus patients [4]. The generalized endotheliopathy induced by COVID-19 disease is assumed to persist even after the infection recovery, which predisposes the patients to increased thrombotic risk [5]. However, our patient also had severe left ventricular dysfunction and thrombophilia mutations which additionally increase the risk for thrombotic complications. Although LV thrombi are present in $10-30 \%$ of patients with dilated 
cardiomyopathy, our patient had multiple thrombi in both ventricles, pulmonary embolism and DVT at the same time which is quite rare and cannot be explained by the presence of left ventricular dysfunction only. The constellation, of endothelial injury, hypercoagulability, and blood stagnation, which are well described previously as Virchow's triad, is responsible for the formation of thrombus [7]. The most logical explanation we have is that post COVID-19, the disease triggered multivascular thrombosis in the patient with coagulation abnormality and previous pneumonia. Left ventricular thrombosis (LVT) complicates both ischemic and non-ischemic cardiomyopathies and is a potential cause of thromboembolic complications such as stroke. Contemporary management of LVT is primarily based on studies before the widespread use of potent pharmacological and interventional therapies such as primary percutaneous coronary intervention. Though advances in imaging techniques have improved detection of LVT, we as clinicians are facing several uncertainties in the management of LVT in our daily practice. Anticoagulation therapy with enoxaparin which was continued with Rivaroxaban leads to decrease of thrombus number and mobility in our patient case. Since our patient had significant improvement of LV function during hospitalization, we assume that heart failure might be caused by COVID-19 induced myocardial injury (myocarditis). Myocarditis may be an important cause of the acute cardiac injury in COVID-19 patients. However, the prevalence, clinical importance, and mechanisms of myocardial inflammation in COVID-19 disease remain unclear. We do not have solid evidence of direct myocardial cytotoxic effects of the virus.

Treatment of these patients is very challenging since due to acute liver injury and reduced AT III levels during the acute phase of the disease, these patients have reduced therapeutic response to heparin. Anticoagulant and concomitant heart failure therapy and close follow up of the patient is mandatory in order to evaluate treatment success and patient recovery [6-8].

One of the limitations in our case is the lack of coronary angiography and cardiac magnetic resonance (CMR) in the acute phase, which are planned to be performed.

\section{Conclusions}

Patients with COVID-19 disease are at increased risk of thromboembolic complications. COVID-19 disease induced inflammation, hypercoagulability and endothelial dysfunction might cause thrombotic complications in any vascular site. We should use high clinical suspicion and imaging techniques for onetime diagnosis and treatment of thrombotic complications in both acute infection and post COVID-19 disease patients.

Funding sources: None.
Authors' contribution: All authors have read and accepted the final version of the manuscript.

Conflict of interests: The authors have no conflict of interest to disclose.

\section{Supplementary material}

The following are the supplementary data related to this article:

Movie 1.2D Transthoracic echocardiography (TTE) four chamber view showing multiple thrombi in the left ventricle with global hypokinesia

Movie 2. 2D Transthoracic echocardiography (TTE) short axis view at the level of mitral valve showing multiple thrombi in the left ventricle with $\mathrm{D}$ shaped left ventricle due to the presence of pulmonary hypertension

Movie 3. 2D Transthoracic echocardiography (TTE) apical three chamber view showing multiple thrombi in the left ventricle with the presence of severe left ventricular dysfunction

Supplementary video related to this article can be found at https://doi.org/10.1556/1647.2021.00047.

\section{REFERENCES}

[1] Behnood B, Mahesh V, Madhavan S, David J, et al.: COVID-19 and thrombotic or thromboembolic disease: implications for prevention, antithrombotic therapy, and follow-up: JACC state-of-the-art review. J Am Coll Cardiol 2020; 75(23): 2950-73.

[2] Poyiadji N, Cormier P, Parth YP, et al.: Acute pulmonary embolism and COVID-19. Radiology 2020; 297(3): 335-8.

[3] Cresti A, Barchitta A, Barbieri A, et al.: Echocardiography and multimodality cardiac imaging in COVID-19. Patients. J Cardiovasc Echogr 2020; 30(2): 18-24.

[4] F. Habash, S. Vallurupalli: Challenges in management of left ventricular thrombus. Ther Adv Cardiovasc Dis 2017 Aug; 11(8): 203-13.

[5] Middeldorp S, Coppens M, Van Haaps TF, et al.: Incidence of venous thromboembolism in hospitalized patients with COVID-19. J Thromb Haemost 2020; 18(8): 1995-2002.

[6] Rosovsky RP, Grodzin C, Channick R: Diagnosis and treatment of pulmonary embolism during the coronavirus disease 2019 pandemic. A position paper from the national PERT consortium. Chest 2020; 158(6): 2590-601.

[7] Ganatra S, Dani SS, Shah S, et al.: Management of cardiovascular disease during coronavirus disease (COVID-19) pandemic. Trends Cardiovasc Med 2020; 30(6): 315-25.

[8] Mehta JL, Calcaterra G, Bassareo PP: COVID-19, thromboembolic risk, and Virchow's triad: lesson from the past. Clin Cardiol 2020 Dec; 43(12): 1362-7.

Open Access. This is an open-access article distributed under the terms of the Creative Commons Attribution-NonCommercial 4.0 International License (https:// creativecommons.org/licenses/by-nc/4.0/), which permits unrestricted use, distribution, and reproduction in any medium for non-commercial purposes, provided the original author and source are credited, a link to the CC License is provided, and changes - if any - are indicated. 